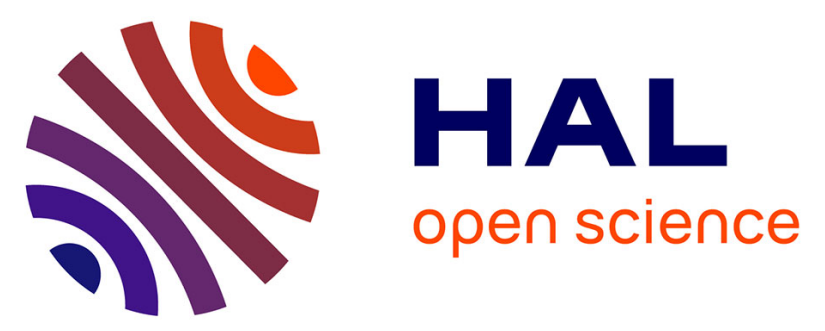

\title{
Investigation of hemicryptophane host-guest binding energies using high-pressure collision induced dissociation in combination with RRKM modeling
}

Parisa Bayat, David Gatineau, Denis Lesage, Vincent Robert, Alexandre Martinez, Richard B Cole

\section{To cite this version:}

Parisa Bayat, David Gatineau, Denis Lesage, Vincent Robert, Alexandre Martinez, et al.. Investigation of hemicryptophane host-guest binding energies using high-pressure collision induced dissociation in combination with RRKM modeling. Journal of The American Society for Mass Spectrometry, 2019, 30 (3), pp.509-518. 10.1007/s13361-018-2109-5 . hal-02323852

\section{HAL Id: hal-02323852 \\ https://hal.science/hal-02323852}

Submitted on 21 Oct 2019

HAL is a multi-disciplinary open access archive for the deposit and dissemination of scientific research documents, whether they are published or not. The documents may come from teaching and research institutions in France or abroad, or from public or private research centers.
L'archive ouverte pluridisciplinaire HAL, est destinée au dépôt et à la diffusion de documents scientifiques de niveau recherche, publiés ou non, émanant des établissements d'enseignement et de recherche français ou étrangers, des laboratoires publics ou privés. 


\section{Investigation of hemicryptophane host-guest binding energies using high-pressure collision induced dissociation in combination with RRKM modeling}

Parisa Bayat,,$^{\S}$ David Gatineau, ${ }^{\S, \dagger}$ Denis Lesage, ${ }^{\S}$ Vincent Robert, ${ }^{*}$ Alexandre Martinez, ' and Richard B. Cole*,

${ }^{\S}$ Sorbonne Université, CNRS, Institut Parisien de Chimie Moléculaire, IPCM, 75005 Paris, France

${ }^{\dagger}$ Univ Grenoble Alpes, CNRS, UMR 5250, DCM, Grenoble, France.

\# Université de Strasbourg, Laboratoire de Chimie Quantique, 1, rue Blaise Pascal, 67008 Strasbourg (France)

" Aix Marseille Université, UMR CNRS 7313-iSm2, Equipe Chirosciences, Av. Escadrille Normandie-Niemen, 13397 Marseille, France

*Correspondence to: Richard B. Cole; e-mail: richard.cole@sorbonne-universite.fr

ABSTRACT. In advancing host-guest $(\mathrm{H}-\mathrm{G})$ chemistry, considerable effort has been spent to synthesize host molecules with specific and well-defined molecular recognition characteristics including selectivity and adjustable affinity. An important step in the process is the characterization of binding strengths of the H-G complexes that is typically performed in solution using NMR or fluorescence. Here, we present a mass spectrometry-based multimodal approach to obtain critical energies of dissociation for two hemicryptophane cages with three biologically-relevant guest molecules. A combination of blackbody infrared radiative dissociation (BIRD) and high-pressure collision induced dissociation (high-pressure CID), along with RRKM modeling, were employed for this purpose. For the two tested hemicryptophane hosts, the cage containing naphthyl linkages exhibited stronger interactions than the cage bearing phenyl linkages. For both cages, the order of guest stability is: choline $>$ acetylcholine $>$ betaine. The information obtained by these types of mass spectrometric studies can provide new insight into the structural features that most influence the stability of $\mathrm{H}-\mathrm{G}$ pairs, thereby providing guidance for future syntheses.

KEYWORDS. Host-Guest chemistry, cryptophanes, blackbody infrared radiative dissociation, collision induced dissociation, MassKinetics 


\section{INTRODUCTION}

Host-guest (H-G) chemistry is a fascinating field of research that involves the establishment of noncovalent interaction(s) between at least two species: the host that is typically a larger molecule containing convergent binding sites which is adapted to accommodate a second species, the guest, containing divergent binding sites [1-3]. This field has found applications in diverse areas of science including molecular recognition [4-6], drug delivery [7-9], catalysis [10-12], bio sensors [13-15], trapping devices [16,17] etc. This very active research topic has employed a wide variety of "cage" architectures as host molecules, and among them are the cryptophane molecules which were introduced by A. Collet in the early 1980s [18]. A typical cryptophane cage consists of two cyclotriveratrylene (CTV) caps, and three linkers which connect these two caps thereby creating a hollow space, or cavity, of defined geometry in between the caps [19]. Since their first synthesis, significant efforts have been focused on modification of cryptophanes in order to design new cavities with novel selectivity characteristics toward various organic and inorganic guest molecules. In extending this approach, an innovative structure called a hemicryptophane was synthesized [20,21]. The structure of a hemicryptophane molecule differs from a cryptophane cage in that one of the CTV caps is replaced with a $\mathrm{C}_{3}$-symmetrical organic group [20]. Hemicryptophanes have been employed for selective inclusion of choline [22], choline phosphate [23] or primary alkylammonium ions [24]. Moreover, hemicryptophanes have been used for enantioselective recognition $[25,26]$ as well as ion-pair recognition [27] and studies pertaining to potential catalytic activity [28] have also been conducted.

H-G chemistry deals with noncovalent interactions which, despite their typically much weaker strength compared to covalent bonds, determine the nature, stability, and preferred 3-D structure(s) of the H-G systems. Therefore, the ability to assess the strengths of the noncovalent binding interactions has a significant importance in characterizing structures of $\mathrm{H}-\mathrm{G}$ complexes, 
and in interpreting their behaviors. Moreover, such assessments can aid in the development of new H-G systems that afford specific desired properties.

So far, most of the H-G chemistry studies of hemicryptophanes have been performed in the condensed phase, and techniques such as fluorescence [23] or nuclear magnetic resonance [29] were primarily used for characterization of $\mathrm{H}-\mathrm{G}$ interactions. However, for a more complete characterization, multiple methods are required. Electrospray ionization mass spectrometry [3032] (ESI-MS), which allows the preservation in the gas phase of weak interactions that existed in the initial solution, is another method of choice to investigate H-G systems. This technique, which offers high sensitivity, short acquisition times, and very low sample consumption, can provide a wealth of information that is sometimes difficult to obtain using other approaches, i.e. acquired results are often complementary to those obtained in the condensed phase.

Investigations of host-guest complexes in the gas-phase [33-37], however, are not so common. Moreover, gas-phase studies have been never performed using hemicryptophane hosts. As noted above, hemicryptophanes can exhibit high selectivity toward various guests. In solution, depending on the size and shape of the host cavity, certain guests can be selectively encapsulated, whereas others may prefer to remain highly solvated and therefore exhibit poor binding characteristics. Gas-phase studies circumvent the problem of solvation/desolvation of the guest and thereby allow examination of the binding affinity in the absence of this parameter. Thus, an added value of gas-phase studies is that they can bring insight into the precise origin of the selectivity (e.g., are there specific intrinsic interaction(s) between host and guest, and does solvation influence the interaction(s)?).

ESI used with single stage MS can provide information pertaining to the stoichiometry of H-G binding, and when combined with tandem mass spectrometry (MS/MS), it can offer a gauge of the strength of the binding interaction(s). Decompositions can be probed using various techniques such as threshold collision induced dissociation (TCID) [38-41], blackbody infrared 
radiative dissociation (BIRD) [42-44], thermal activation in a quadrupole ion trap [45-47], collisional activation in a Fourier transform ion cyclotron resonance (FT-ICR) [48-50] instrument, among others.

In this manuscript, two hemicryptophane cages $\mathbf{1}$ and $\mathbf{2}$ (Figure 1) are employed as the host molecules and their binding properties towards three biologically important guests: choline $(\mathbf{C})$ [51], acetylcholine (AC) [52] and betaine (B) [53,54] (Figure 1) are studied in the gas phase. When utilizing tandem mass spectrometry techniques for comparison purposes, commonly survival yield (SY) is plotted as a function of energy in the center of mass frame. The so-called "SY curves" generated in this manner have sigmoidal shapes, and their inflection points are usually used to compare the relative stabilities of a series of $H-G$ complexes [33-37,55]. There are various parameters influencing the position of the SY curves such as: the number of collisions (which depends on the size of the ion and the target gas pressure), critical energy, entropy of activation, and available time for decomposition that all combine to determine the "kinetic shift" [56,57] (i.e., the excess energy that needs to be applied to the precursor ion, relative to its critical energy of dissociation, in order to observe fragmentation in the time scale of mass spectrometric detection). By using energy in the center of mass frame of reference, the effect of the changing mass of the ions can be taken into account. However, there is a need for a means to more thoroughly consider all of the above-mentioned parameters that influence the kinetic shift. Here, we present an approach that allows the comparison of the binding strengths of different $\mathrm{H}-\mathrm{G}$ pairs using a combination of BIRD and high-pressure CID mass spectrometric techniques, combined with Rice-Ramsperger-Kassel-Marcus (RRKM) [58-61] modeling. Using this method which allows absolute energy measurements, the effect of all the implicated parameters is included in the final reported bond dissociation energies. Thus, the main goal of this study pertaining to "method development" is to present a method that can be used to obtain intrinsic binding energies in the absence of complicating effects of counter ion or solvent. 
Relevant parameters related to binding strength, such as critical energy $\left(E_{0}\right)$, activation energy $\left(E_{a}\right)$ are defined in Table $\mathrm{S} 1$ of the supporting information.

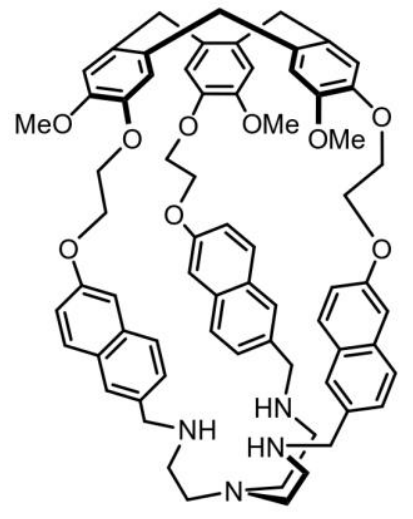

1

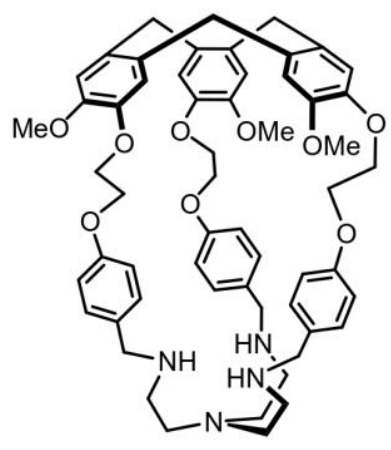

2

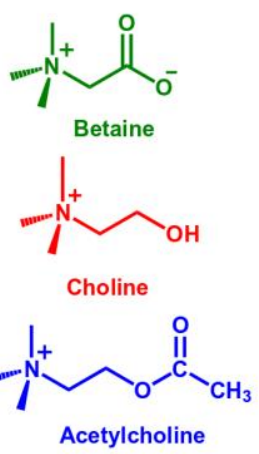

Figure 1. Structures of hemicryptophane cages 1 and 2; and studied guests.

\section{EXPERIMENTAL}

Two hemicryptophane cages, $\mathbf{1}$ and $\mathbf{2}$ were synthesized according to previously published methods [62,63] and their stock solutions $(1 \mathrm{mM})$ were made in dichloromethane. Stock solutions of choline, acetylcholine and betaine were prepared in methanol $(1 \mathrm{mM})$ and individual working solutions were prepared by diluting the host and each individual guest molecule $(1: 1)$ in methanol. For experiments performed on FTICR and timsTOF instruments, concentrations of both host and guest were $10^{-6} \mathrm{M}$, and for those performed on the Quattro II, the concentrations of both partners were $10^{-4} \mathrm{M}$.

A 'Quattro II' triple quadrupole mass spectrometer (Waters, Manchester, UK) equipped with an electrospray ionization source (ESI) was used to obtain MS and MS/MS spectra. The main reason that the triple quadrupole instrument was selected for high-pressure CID experiments is that it minimizes discrimination between low and high mass ions. A capillary potential of 3.5 $\mathrm{kV}$, cone voltage of $50 \mathrm{~V}$, and extractor potential of $6 \mathrm{~V}$ were used. The source block temperature was kept at $100{ }^{\circ} \mathrm{C}$. Nitrogen was used as nebulizing and drying gas at $20{ }^{\circ} \mathrm{C}$. 
Two different techniques: high-pressure CID and low-pressure threshold CID (TCID) were employed in this work. For high-pressure CID experiments, argon $\left(5 \times 10^{-3}\right.$ mbar $)$ was used as collision gas, and for optimized transmission of ions, the following settings were used: for the first quadrupole: ion energy of $0 \mathrm{eV}$, lens 5 was at $100 \mathrm{~V}$, and lens 6 at $0 \mathrm{~V}$; and for the second quadrupole: ion energy of $10 \mathrm{eV}$, lens 7 was at $250 \mathrm{~V}$, lens 8 at $175 \mathrm{~V}$, and lens 9 at $50 \mathrm{~V}$.

For TCID experiments, xenon $\left(10^{-5} \mathrm{mbar}\right)$ was employed as collision gas and the following settings were utilized for efficient ion transmission: for the first quadrupole: ion energy of $0 \mathrm{eV}$, lens 5 at $100 \mathrm{~V}$ and lens 6 at $0 \mathrm{~V}$; and for the second quadrupole in the QhQ instrument: ion energy of $0 \mathrm{eV}$, lens 7 at $250 \mathrm{~V}$, lens 8 at $10 \mathrm{~V}$ and lens 9 at $0 \mathrm{~V}$.

Collision gas was introduced into the collision cell using a customized line as previously described [64]. Masslynx V4.0 sp2 software was used for analyzing the MS and CID tandem mass spectra. Breakdown curves were obtained by plotting survival yield $\left(S Y=I_{P} /\left(I_{P}+\sum I_{F}\right)\right)$, where $I_{P}$ is the intensity of the precursor ion, and $I_{F}$ is that of the fragment ion(s), versus collision energy in the laboratory frame of reference, $E_{l a b}$.

Ion mobility experiments were performed using a trapped ion mobility mass spectrometer (timsTOF) instrument (Bruker Daltonics, Bremen, Germany). Spectra were obtained using an electrospray ionization source operating in positive ion mode. Solutions were injected at a flow rate of $5 \mu \mathrm{Lmin}^{-1}$. Capillary voltage was set at $3.6 \mathrm{kV}$, end plate offset at $500 \mathrm{~V}$, and nebulizer gas pressure at $4.4 \mathrm{psi}$. The drying gas temperature was set at $200{ }^{\circ} \mathrm{C}$, and its flow rate was 3 $\mathrm{Lmin}^{-1}$. Ion mobility separations were first performed in the inverse reduced mobility $\left(1 / K_{0}\right)$ range of 0.77 to $1.93 \mathrm{Vscm}^{-2}$, and then, the spectra were recorded again in a narrowed inverse mobility range depending on the $1 / K_{0}$ values of the ions of interest. Nitrogen was used as buffer gas at a temperature of $27^{\circ} \mathrm{C}$. Accumulation time for a single analysis was set at $10 \mathrm{~ms}$, and the spectra were recorded for 1 minute. 
BIRD experiments were performed using a $7 \mathrm{~T}$ hybrid quadrupole-fourier transform ion cyclotron resonance (FT-ICR) mass spectrometer (ApexQe, Bruker Daltonics, Billerica, USA). Solutions were injected at a flow rate of $120 \mu \mathrm{L} \cdot \mathrm{h}^{-1}$ into an Apollo II electrospray ionization source with $\mathrm{N}_{2}$ as nebulizing gas. The temperature of the source was set at $250{ }^{\circ} \mathrm{C}$, ESI voltage at $4500 \mathrm{~V}$, capillary exit at $300 \mathrm{~V}$, skimmer I at $150 \mathrm{~V}$, and skimmer II at $8 \mathrm{~V}$. A resistiveheating metallic "ribbon" was installed external to the FT-ICR cell, covering the entire cell length. An accuracy check of the cell temperature was performed using dissociation of the sodiated dimer of leucine enkephalin, with known activation parameters [65]. A temperature probe was also placed between the metallic ribbon and the cell housing; its displayed temperature showed a maximum difference of $3{ }^{\circ} \mathrm{C}$ compared to the temperature obtained by calibration. All spectra were acquired using XMASS (version 6.1, Bruker Daltonics) in broadband mode with the number of data points set at $512 \mathrm{~K}$.

\section{MODELING DETAILS}

Vibrational frequencies of the precursor ions were calculated using the GAMESS $[66,67]$ computational package employing the Hartree-Fock method and Slater-type basis sets. These vibrational frequencies have been used as a necessary input for RRKM calculations. Although this level of theory is relatively low, it has been shown $[68,69]$ that RRKM calculations are not sensitive to the vibrational frequencies of the initial state when they are not compared with those of the transition state. CID experiments were simulated using MassKinetics Scientific Demo software (version 1.17.2.648 Beta, provided courtesy of Prof. László Drahos) [70]. Calculations were performed within the RRKM formalism [58-61]. To simulate internal energy distributions and their change with time, a model based on a truncated Maxwell-Boltzmann distribution of internal energy and characteristic temperature $\left(T_{\text {char }}\right)$ was utilized. $T_{\text {char }}$ is the temperature of the Boltzmann distribution of internal energies of the ions before decomposition (see Supporting 
Information and Figure S1 for more detail) [64,71-73]. As has been evaluated previously [64], and considering that precursor ions undergo several hundred collisions, leading to rather long residence times inside the collision cell [74,75], a flight time of $0.002 \mathrm{~s}$ has been used. It should be noted that because we are relying upon a reference ion for calibration of mean internal energy, the input flight time has a minimal effect on the calculated critical energies. Instead, it mainly influences the initial temperature of the reference ions, and as a consequence, that of the studied complexes. According to this model, activation and dissociation steps are discrete and consecutive. In other words, in a first step, a voltage is applied that results in acceleration of the ions and collision with the target gas (Ar). In this process, kinetic energy is converted to internal energy and ions become activated. Afterwards, in a second step, dissociation of the activated molecular ions occurs. One of the $\mathrm{H}-\mathrm{G}$ complexes that was amenable to the BIRD technique, namely $[(\mathbf{B}+\mathbf{2})+\mathrm{H}]^{+}$, was selected as the reference ion. For this complex, the mean internal energy $<E_{\text {int }}>$ and $T_{\text {char }}$ corresponding to each point of its $S Y$ curve were calculated. Then, a linear relationship between the CID collision energy $\left(E_{\text {lab }}\right)$ and $\left\langle E_{\text {int }}>\right.$ was established $[64,71-$ 73]. In the next step, this calibration was used for all $\mathrm{H}-\mathrm{G}$ pairs and $S Y$ curves were simulated and fitted to experimental data to obtain the critical energy of dissociation for each complex. This model is only valid for CID experiments conducted at high pressures under analytical conditions [64]

In order to extract critical energy $\left(E_{0}\right)$ from the Arrhenius plot (obtained using BIRD experiments, Figure S2), MassKinetics [70] Scientific software (version 1.17.2.648 Beta) was utilized. For this purpose, calculations were performed considering that ions remain in thermal equilibrium during their fragmentation. Using this model, critical energies were adjusted until the best fit between the experimentally obtained Arrhenius plots and the calculated ones was achieved. It should be noted that the critical energy is defined as the potential energy difference between the transition state (in its ground state) and the precursor ion (in its ground state) at $0 \mathrm{~K}$. 
The activation energy $\left(E_{a}\right)$ is defined as the difference between the internal energy content of the transition state and that of the precursor ion at the temperature of the experiment, and is defined only for thermal reactions. The connection between these two quantities is explained elsewhere [42].

\section{RESULTS AND DISCUSSION}

Both hemicryptophane cages 1 and 2 (Figure 1), that serve as host molecules in this study, contain a cyclotriveratrylene (CTV) unit and a tris(2-aminoethyl)-amine (tren) group as their two caps. But they can be distinguished by the nature of their linkers: naphthyl (cage 1) versus phenyl (cage 2) groups. Thus, when comparing the behaviors of guest molecules, any differences in the binding strengths of their H-G complexes can be directly attributed to the characteristics of the linkers and the latter's influence on the final complex structure. Three biologically relevant molecules: betaine (B) [53,54], choline (C) [51], and acetylcholine (AC) [52], each containing a permanently charged trimethylammonium substituent, were selected as guest molecules for this study. Betaine is zwitterionic in neutral solution, and it should be noted that some other guests such as glycine, $\beta$-alanine and taurine were also tried, but no evidence of H-G complexes with cage $\mathbf{1}$ or cage $\mathbf{2}$ were observed for these latter three compounds in obtained mass spectra.

When a 1:1 solution of $H: G$ is analyzed by ESI-MS, in the case of choline and acetylcholine, the observed complex is $[\text { Host }+ \text { Guest }]^{+}$, whereas for zwitterionic betaine, $[(\text {Host }+ \text { Guest })+H]^{+}$is observed. Thus, the betaine complex requires a proton to create the singly charged cationic species observed in the mass spectrum. Under CID conditions, AC and $\mathbf{C}$ complexes with either cage dissociate by loss of the cage, and therefore, their major fragments are the guest molecules bearing the charge. By contrast, protonated $\mathbf{B}$ complexes formed with either cage dissociate by loss of the neutral guest, thus protonated cages are the observed fragments. It should be noted 
that in the case of acetylcholine H-G complexes, a very low abundance of a fragment ion at $\mathrm{m} / \mathrm{z}$ 87.0 was also observed which is produced by loss of the $-\mathbf{N}(\mathbf{M e})_{3}$ group from acetylcholine. The abundance of this fragment ion was taken into account in the survival yield calculations. The masses of the different complex ions and their fragments are summarized in Table S2, and their CID spectra are shown in Figure S3.

In this study, our goal is to compare the critical energies for dissociation of the various observed H-G complexes. To this end, the internal energy uptake occurring in high-pressure CID experiments must be calibrated using a reference molecule that has: 1) accurately known activation parameters, and 2) a structure and number of degrees of freedom (DOF) quite similar to those of the systems under study. If this latter condition is met, the amount of energy deposited should be almost the same in all cases. Previous studies [64,71-73] revealed that the mean internal energy $\left\langle E_{\text {int }}>\right.$ varies only slightly with the number of DOF and with a change in the identity of the precursor ion, thereby giving us confidence that the above precautions are sufficient to obtain reasonable and trustworthy results.

In the next step, the use of a reliable method to calculate Arrhenius parameters for the reference compound is required. Among the developed tandem mass spectrometry methods, one of the most established ones that can directly give activation parameters is BIRD [42]. In practice, only precursor ions which are fragile enough to produce sufficiently abundant fragment ions under the accessible temperature range of the BIRD set-up (300 to $410 \mathrm{~K}$ in our instrument) are amenable to this technique. In this investigation, among all the studied H-G complexes, $[(\mathbf{B}+\mathbf{2})+\mathrm{H}]^{+}$most readily fulfilled this condition. A detailed explanation of the BIRD experiments performed on this ion is presented in the Supporting Information, from which an activation energy $\left(E_{a}\right)$ of $1.27 \pm 0.05 \mathrm{eV}$ and the logarithm of pre-exponential factor $(\log A)$ of $14.2 \pm 0.6$ were obtained for $[(\mathbf{B}+\mathbf{2})+\mathrm{H}]^{+}$complex. 
It should be noted that BIRD experiments were performed on all of the $\mathrm{H}-\mathrm{G}$ complexes under study, but limitations were found. For example, $[\mathbf{C}+\mathbf{1}]^{+},[\mathbf{C}+\mathbf{2}]^{+}$and $[\mathbf{A C}+\mathbf{1}]^{+}$complexes did not dissociate in the available temperature range, and $[(\mathbf{B}+\mathbf{1})+\mathrm{H}]^{+}$produced only a low abundance of fragment ions. Moreover, in this case, we were apprehensive about the presence of an interfering ion (Figure S4). Therefore, it was not possible to construct reliable kinetic plots for this complex. In the case of $[\mathbf{A C}+\mathbf{2}]^{+}$, fragment ions were produced in low abundance, and the associated kinetic plots are not quite linear $\left(\mathrm{R}^{2}=0.9740\right.$, Figure S5). Moreover, the obtained activation parameters for $[\mathbf{A C}+2]^{+}\left(E_{a}\right.$ of $1.33( \pm 0.07) \mathrm{eV}$ and $\log A$ of $13.8( \pm 0.9)$, SI Figure S5), include more uncertainty as compared to those of the $[(\mathbf{B}+\mathbf{2})+\mathrm{H}]^{+}$complex. Because of the higher quality BIRD data obtained for the $[(\mathbf{B}+\mathbf{2})+\mathrm{H}]^{+}$complex, it was selected as the reference precursor for the calibration of mean internal energy $\left(\left\langle E_{\text {int }}>\right)\right.$ of ions undergoing high-pressure CID.

BIRD experiments can provide the activation energy of the reference $\mathrm{H}-\mathrm{G}$ complex; however, for calibration of the mean internal energy using RRKM modeling, the critical energy of this reference ion is required. In order to calculate the critical energy, one needs to have precise information about the transition state. However, for a large system such as the $[(\mathbf{B}+\mathbf{2})+\mathrm{H}]^{+}$ complex in which an ensemble of non-covalent interactions is responsible for keeping host and guest molecules together, it is very difficult to calculate the transition state. For this reason, the experimentally measured pre-exponential factor $(\log A=14.2( \pm 0.6)$ is used in the MassKinetics software. Then, considering a thermally-equilibrated system, critical energies were adjusted until the best fit between the experimentally obtained Arrhenius plot and the calculated one was achieved (Figure S2). By doing so, an $E_{0}$ of $1.19 \mathrm{eV}$ was gleaned for the reference system.

For all H-G pairs, survival yield curves were obtained as a function of collision energy in the laboratory frame of reference using high-pressure CID (Figure 2). Afterwards, a model based upon a truncated Maxwell-Boltzmann distribution was used to calculate the deposited internal 
energy and its evolution with time. In previous studies, it was demonstrated that this model is suitable for simulating fragmentation occurring under analytical conditions, including high collision gas pressures (multiple collision regime) [64,71-73].

For the reference complex, $[(\mathbf{B}+\mathbf{2})+\mathrm{H}]^{+}$, RRKM modeling allows calculation of the mean internal energy before decomposition $\left(<E_{\text {int }}>\right)$ corresponding to each collision energy, and Figure $2 \mathrm{~b}$ illustrates the plot of calculated $\left\langle E_{\text {int }}\right\rangle$ as a function of $E_{\text {lab. }}$. The extrapolated intercept of $3.46 \mathrm{eV}$ indicates the initial mean internal energy of the ions, and corresponds to a characteristic temperature of $386 \mathrm{~K}$ for the reference molecule which is a little bit higher than that of the electrospray source block $(373 \mathrm{~K})$. This higher temperature can be attributed to collisions that ions may undergo during their acceleration from the ionization source towards the collision cell. 

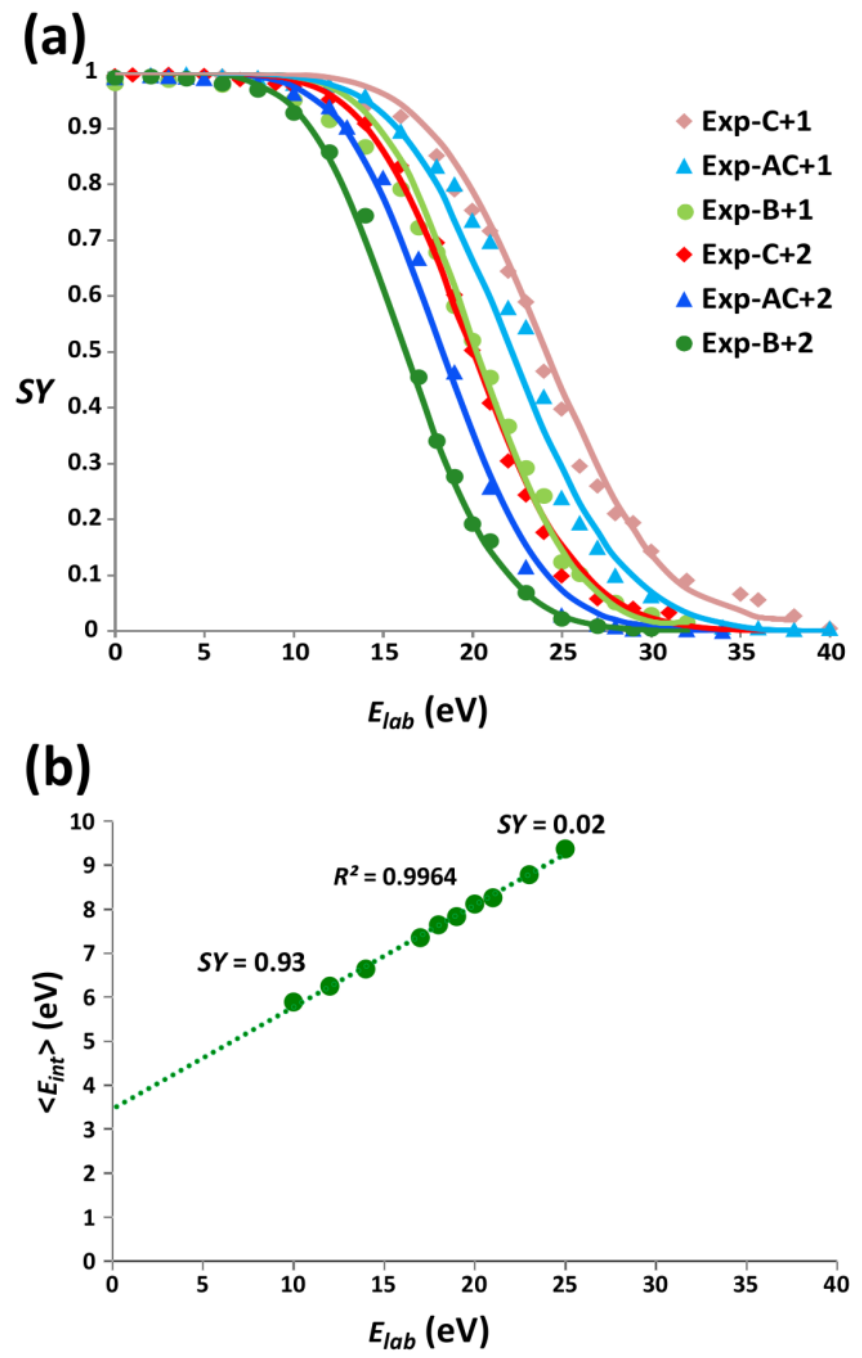

Figure 2. (a) Experimental (markers) and theoretical (lines) $S Y$ curves obtained using highpressure CID for various H-G complexes studied in this project; (b) plot of calculated mean internal energy versus energy in the laboratory frame of reference for $[(\mathbf{B}+\mathbf{2})+\mathrm{H}]^{+}$.

The equation $\left.\left(<E_{\text {int }}\right\rangle=0.2317 \times E_{\text {lab }}+3.4614\right)$ is employed to gauge deposited mean internal energy for other H-G pairs by replacing its intercept with the mean internal energy of each H-G complex at the initial temperature $(386 \mathrm{~K})$. Because all of the $\mathrm{H}-\mathrm{G}$ complexes are characterized by similar sizes and similar types of interactions, a pre-exponential factor of 14.2 (that of the reference $[(\mathbf{B}+\mathbf{2})+\mathrm{H}]^{+}$) is used for all cases (in BIRD experiments performed on the $[\mathbf{A C}+\mathbf{2}]^{+}$ complex, a very similar value for the pre-exponential factor, i.e., $\log A$ of $13.8( \pm 0.9)$, was obtained). Afterwards, using this constant pre-exponential factor, critical energies were adjusted manually until the theoretical $S Y$ curves gave the best fit to the experimental ones. 
Figure 2a displays the experimental (shown by data points) and calculated $S Y$ curves obtained for all six H-G complexes. In each case, an acceptable fit could be established between the two sets of data. It should be noted that for $[\mathbf{C}+\mathbf{1}]^{+}$, because of the presence of an interfering isobaric ion (Figure S6), the highest energy part of the $S Y$ curve could not be reliably obtained experimentally. For the beginning portion of the $S Y$ curve for $[\mathbf{C}+\mathbf{1}]^{+}$, the contribution of this isobaric ion's abundance was removed from the abundance of the precursor ion (based upon the $\%$ of interfering ion observed in the ESI-MS spectrum and known isotopic abundances). The obtained critical energies for the studied H-G pairs are summarized in Table 1.

Table 1 reveals that the $E_{0}$ values fall within a rather narrow range for all ions; nonetheless, some informative trends and differences can be extracted from the data. For instance, complexes of cage 1 exhibit higher critical energies, and thus are all more stable than those of cage $\mathbf{2}$. This trend establishes that the linkers (the only difference between cage $\mathbf{1}$ and cage $\mathbf{2}$ host molecules) play important roles in determining the strength of the H-G complexes. Another interesting comparison is the relative stability ranking between various guests for a given host cage. Notably, for both cages $\mathbf{1}$ and $\mathbf{2}$, the order of stability of H-G complexes is as follows: choline > acetylcholine $>$ betaine.

Table 1. Critical energies for dissociation of $H-G$ complexes studied in this project.

\begin{tabular}{llll}
\hline Entry & Complex & $E_{0}(\mathrm{eV})(\mathrm{RRKM}$ modeling $)$ & $E_{a}(\mathrm{eV})$ and $\log A(\mathrm{BIRD})$ \\
\hline 1 & {$[\mathbf{C}+\mathbf{1}]^{+}$} & $1.31( \pm 0.08)^{*}$ & --- \\
2 & {$[\mathbf{A C}+\mathbf{1}]^{+}$} & $1.29( \pm 0.08)$ & --- \\
3 & {$[(\mathbf{B}+\mathbf{1})+\mathrm{H}]^{+}$} & $1.23( \pm 0.07)$ & --- \\
4 & {$[\mathbf{C}+\mathbf{2}]^{+}$} & $1.27( \pm 0.08)$ & --- \\
5 & {$[\mathbf{A C}+\mathbf{2}]^{+}$} & $1.24( \pm 0.07)$ & $E_{a}=1.33( \pm 0.07)^{*}$, \\
6 & & & $\log A=13.8( \pm 0.9)$ \\
& {$[(\mathbf{B}+\mathbf{2})+\mathrm{H}]^{+}$} & $1.19( \pm 0.05)$ & $E_{a}=1.27( \pm 0.05)$, \\
& & & $\log A=14.2( \pm 0.6)$
\end{tabular}

*See Supporting Information for details concerning calculation of uncertainty values. 
Although the obtained critical energies do not differ tremendously from one another in terms of absolute values, as far as relative values are concerned, they are entirely consistent with the BIRD results. In BIRD experiments, it was observed that $[\mathbf{C}+\mathbf{1}]^{+},[\mathbf{C}+\mathbf{2}]^{+}$and $[\mathbf{A C}+\mathbf{1}]^{+}($the three complexes with the highest $E_{0}$ values) did not dissociate in the accessible temperature range. Moreover, weaker binding $[\mathbf{A C}+\mathbf{2}]^{+}$and $[(\mathbf{B}+\mathbf{1})+\mathrm{H}]^{+}$complexes did undergo dissociation, but they did not produce sufficient abundances of fragment ions to obtain highly linear kinetic plots. Finally, the least stable $[(\mathbf{B}+\mathbf{2})+\mathrm{H}]^{+}$complex did produce enough fragment ions to enable obtainment of linear kinetic and Arrhenius plots for this complex. Considering the different behavior of these complexes, it is evident that when the upper limit of attainable temperature in BIRD experiments is not extremely high, then, minor differences in critical energies become important. Thus, small differences in the guests' functional groups, or identity of the linkers in the cages, can result in different stabilities, thereby enabling sufficient dissociation for one complex, and not enough dissociation for others in BIRD spectra.

In order to get additional, complementary information about the $\mathrm{H}-\mathrm{G}$ systems, TCID experiments were performed. In these experiments, the pressure of the collision gas (Xe) was held at a low value $\left(10^{-5}\right.$ mbar $)$ where single collision conditions predominate. In order to take into account the effect of the variable sizes of the H-G complexes, normalized abundances of the ions $\left(I_{F} /\left(I_{P}+\sum I_{F}\right)\right)$ were plotted as a function of the center of mass energy $\left(E_{c . m}\right)$. Center of mass energy is the maximum energy that can be transferred during one collision and is given by $\left(E_{c . m .}=E_{\text {lab }} \times m_{\text {gas }} /\left(m_{\text {gas }}+M_{i o n}\right)\right)$ where $m_{\text {gas }}$ is the mass of the collision gas and $M_{\text {ion }}$ is the mass of the precursor ion [76]. TCID plots are presented in Figure 3. 


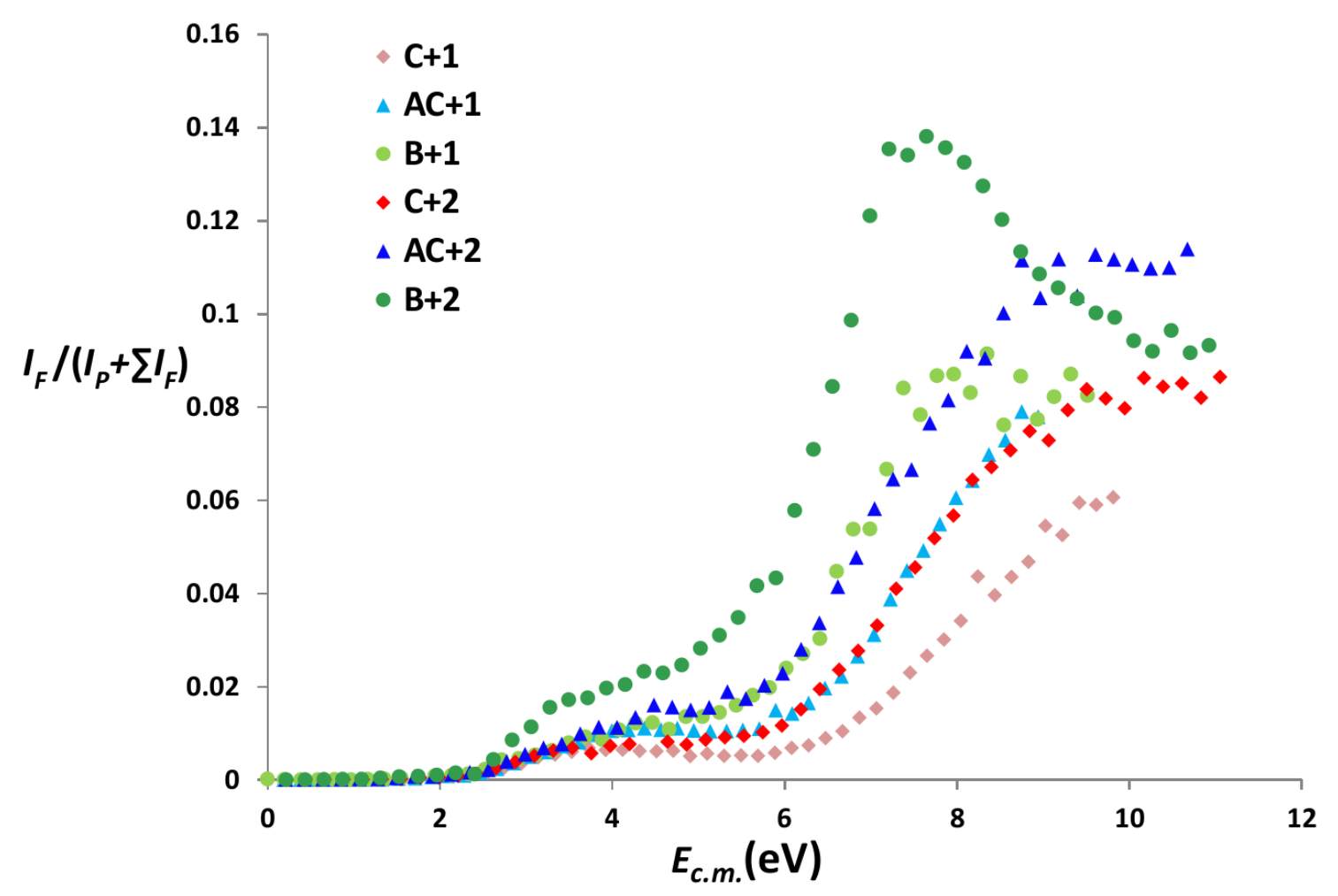

Figure 3. Normalized intensity of the fragment ion vs. collision energy (center of mass frame) for dissociation of the six $\mathrm{H}-\mathrm{G}$ pairs studied in this project using TCID.

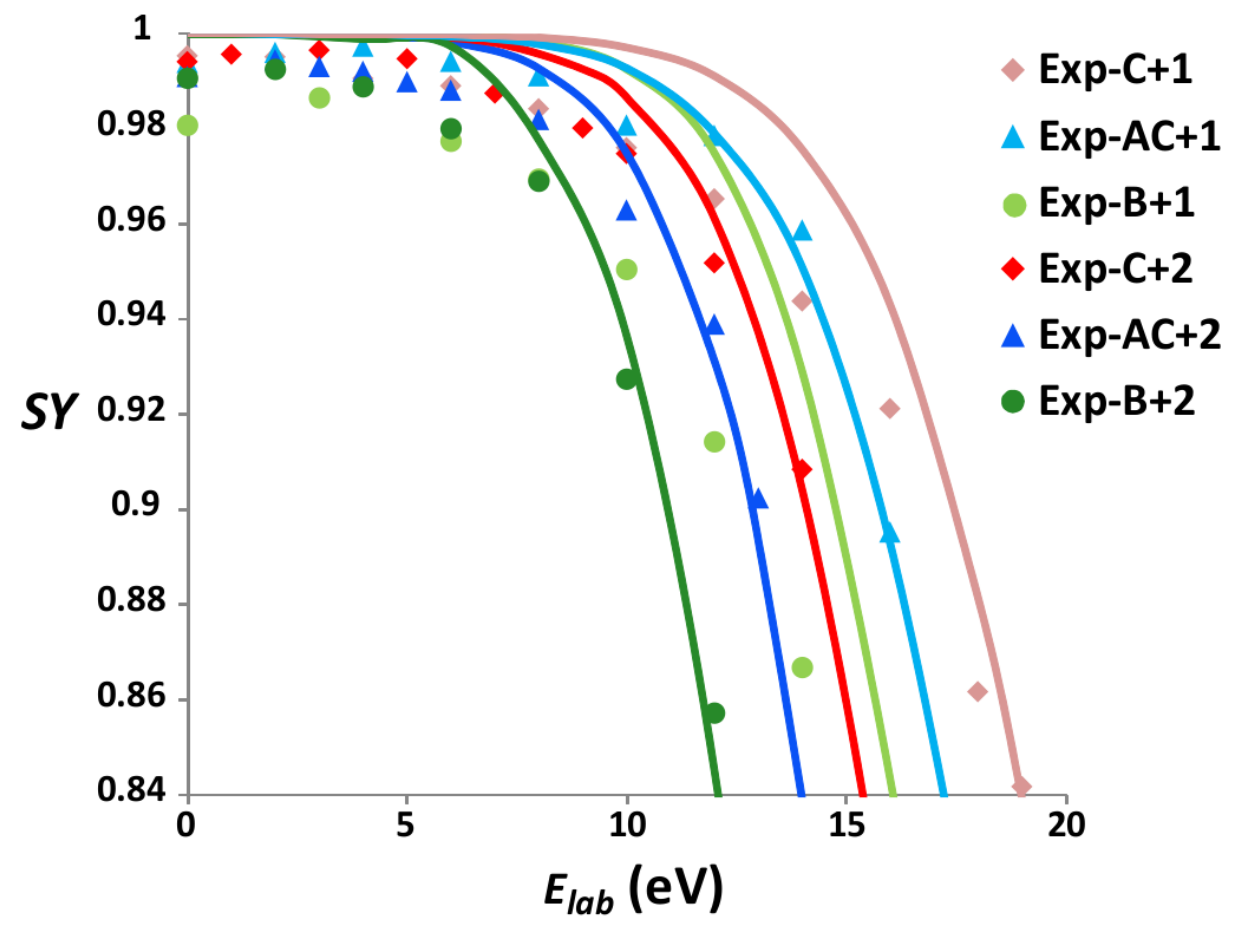

Figure 4. Enlargement of the beginning part of the experimental (data points) and theoretical $S Y$ curves of the H-G complexes studied by high-pressure CID in this project (the inconsistency between theory and experiment at the first part of the curve is postulated to be due to a minor quantity of fragile $\mathrm{H}-\mathrm{G}$ complex (likely exclusion complexes) in each case). 
The most interesting feature revealed in Figure 3 is that there are two separate decomposition steps in each curve. But at the same time, the complication of having two steps makes it difficult to perform RRKM calculations on them. This evidence of a two-step process can be rationalized by considering that two types of structures (conformers) exist for the H-G complexes. The first step of dissociation corresponds to easily dissociable, very fragile structures, whereas the second step pertains to complexes with higher stability. In TCID, only a small percentage of the ion population undergoes dissociation, and the exact ratio of abundances of the two different species is not directly deducible from the curves. However, in high-pressure CID, the entire population undergoes dissociation, and this ratio can be obtained by direct comparison of the normalized intensities. Close inspection of the beginning (left side) portion of the $S Y$ curves (Figure 4), where the survival yield first begins to drop at extremely low collision energy values, reveals the presence of a minor amount of readily dissociating, weakly bound species. Notably, this weakly bound conformer has completely dissociated before dissociation of the higher abundance, more strongly bound H-G complexes begins. These data obtained by high-pressure CID corroborate the TCID data, and prompt us to postulate that the former weakly bound complexes correspond to exclusion complexes (guests exhibiting weak binding to the exterior of the host cages), whereas the more strongly bound conformers represent the expected inclusion complexes (guests located in, and exhibiting binding to, the interior of the host cage). Since the weakly bound species constitute only a small portion of the population, their presence will not lead to significant errors in the above calculations and the obtained critical energies. It should be noted that in the BIRD experiments, it is highly probable that these fragile complexes are dissociated either during the hexapole trapping step (collision with Ar and storage time on the order of one second) before being sent to the ICR cell, or upon rf isolation inside the ICR cell, and that is why the presence of this easily dissociating population is not evident in the BIRD experiments; but, this trapping step does not exist in CID experiments. 
The most fragile complexes, likely corresponding to exclusion complexes, are characterized by a small number of non-covalent interactions between host and guest molecules. $E_{0}$ values shown in Table 1 correspond to more tightly bound $\mathrm{H}-\mathrm{G}$ complexes that are characterized by a larger ensemble of non-covalent interactions. This stronger interaction is consistent with a placement of guests inside the host molecules, in the form of inclusion complexes. Corroboration for this statement comes from the use of glycine as a guest molecule. Glycine is very similar to betaine except that, instead of the -NMes group of betaine, there is a $-\mathbf{N H}_{3}$ group on glycine. For the glycine guest, however, no H-G complex was formed with either of the two cage molecules. This contrast in behavior indicates that the presence of the cationic - $\mathbf{N M e}_{3}$ group plays a critical role in stabilizing the $\mathrm{H}-\mathrm{G}$ complexes for the betaine guest, likely through interaction with the CTV cap. Further evidence for the simultaneous existence of (minor) exclusion complexes and (predominant) inclusion complexes comes from ion mobility experiments performed on the $6 \mathrm{H}-\mathrm{G}$ complexes. While one prevalent structure is observed for each complex (Figure 5, Figure S7), a small quantity of a second conformer with larger collision cross section is evident in several of the mobilograms. A larger collision cross section would be expected, of course, for an exclusion complex conformation. The presence of this small quantity of $\mathrm{H}-\mathrm{G}$ species exhibiting somewhat larger collision cross sections than the dominant $\mathrm{H}-\mathrm{G}$ species (see Figure 5) is entirely consistent with the co-existence of very minor amounts of exclusion complexes in the presence of highly prevalent inclusion complexes. Most important to note is that the energies of dissociation of $\mathrm{H}-\mathrm{G}$ complexes obtained using BIRD or high-pressure CID in combination with RRKM modeling correspond to the dominant species in the ion population. 


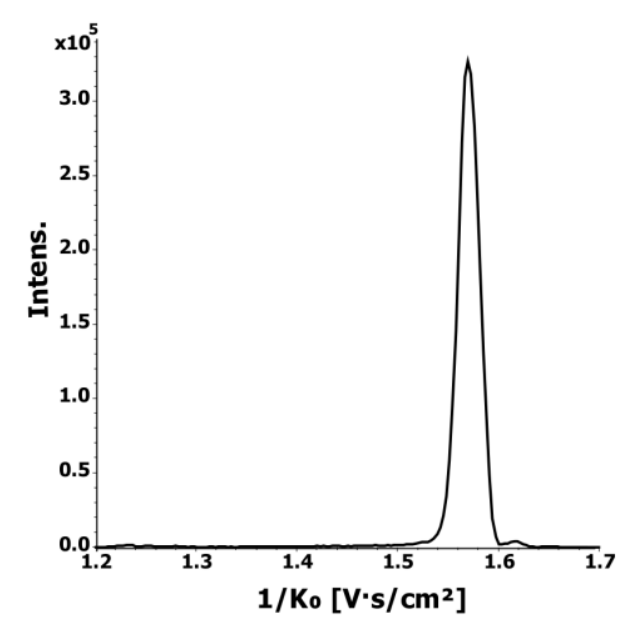

Figure 5. Extracted ion mobilogram of $[(\mathbf{B}+\mathbf{1})+\mathrm{H}]^{+}$precursor ion showing principal peak plus small peak (to the right) corresponding to second conformer of lower mobility and higher collision cross section.

\section{CONCLUSIONS}

In this manuscript, a multimodal approach was employed using the established BIRD technique in combination with $S Y$ curves obtained by high-pressure CID, and RRKM modeling to measure critical energies of a series of H-G complexes. The activation energy and preexponential factor of the reference complex, $[(\mathbf{B}+\mathbf{2})+\mathrm{H}]^{+}$, measured using BIRD, an absolute energy evaluation technique, were used to calibrate the internal energy distributions of the other H-G complexes during high-pressure CID experiments. Afterwards, by making the supposition that the transition entropy for decompositions of all complexes is approximately equal, critical energies were calculated for all the H-G pairs. This simple approach presents a highly facile, and at the same time reliable, method for ranking the stabilities of $\mathrm{H}-\mathrm{G}$ complexes. However, one limitation of this technique is that it is mainly applicable to H-G systems that are characterized by a similar size.

Obtained critical energies display two trends in the stabilities of H-G complexes: first, cage 1 containing a naphthyl linker makes more stable complexes with all three guests compared to cage 2 bearing a phenyl linker; second, the order of the stability of H-G pairs is choline > acetylcholine $>$ betaine for both cage $\mathbf{1}$ and cage 2 . These results can be compared to previous 
solution-phase results from three different perspectives. First, gas-phase results obtained for hemicryptophane 2 with choline and betaine guest molecules are consistent with those observed in solution [22]. Employing a very similar hemicryptophane cage that is water soluble, a selective recognition towards choline versus betaine was observed in solution [22]. The gasphase binding energies that we obtained show that the cage has an intrinsically stronger interaction with choline than with betaine. Therefore, the complementary information provided by the gas-phase studies allows us to conclude that the lower observed affinity of betaine is not attributable solely to its higher solvation in water versus that of choline.

There is a second piece of information that can be extracted from the obtained gas-phase data that is complementary to solution-phase data. It has been shown previously using NMR spectroscopy [24] that cage 2 has a strong affinity to primary alkylammonium cations. In the current study, when we used glycine as a guest molecule, despite the presence of a primary alkylammonium group, no H-G complex was formed with either of the two cages. This contrasting behavior adds the complementary new information that both sides of the guest (not just the primary alkylammonium end) play crucial roles in the formation of $\mathrm{H}-\mathrm{G}$ inclusion complexes.

A third piece of complementary information that the current study provides is the ranking of different functional groups for their abilities to engage in strong non-covalent interactions with the two cages in the absence of solvent and counter ion. This was achieved by comparing three guest molecules, all having the same trimethylammonium group on one end, and dissimilar functional groups on their other end. It was observed that the alcohol functional group has a stronger interaction with both cages than the acetyl group, and the latter is held more strongly than the carboxylate group.

Additionally, TCID experimental results lead to the deduction of the presence of two types of structures for each H-G complex. However, ion mobility experiments on the $6 \mathrm{H}-\mathrm{G}$ complexes 
showed one predominant structure in all cases, although a small quantity of a second structure (exhibiting a higher CCS) was evident in some of the mobilograms. We are confident that the obtained energies using BIRD or high-pressure CID in combination with RRKM modeling correspond to the dominant species in the ion population, that we postulate to be an inclusion complex. The minor second conformer that was most readily visible in TCID experiments likely corresponds to an exclusion complex.

The complementary techniques employed in this manuscript are useful not only for estimation of bond dissociation energies, but also for accurate surveying of the influence of different functional groups on $\mathrm{H}-\mathrm{G}$ binding properties. Utilization of this approach can provide a wealth of information pertinent to chemists who seek to design new H-G systems with well-honed properties.

\section{ACKNOWLEDGMENTS}

PB acknowledges financial support from a Bourse Ministérielle awarded by the French government. DG acknowledges support from the Arcane Labex (ANR-11-LABX-0003-01). Financial support from the French National FT-ICR network (FR 3624 CNRS), and the MetaboHUB, ANR-11-INBS-0010 grant are gratefully acknowledged.

\section{REFERENCES}

1. Vincenti, M. Host-guest chemistry in the mass spectrometer. J. Mass Spectrom. 30, 925939 (1995).

2. J. Cram, D. \& M. Cram, J. Container molecules and their guests. (The Royal Society of Chemistry, 1997).

3. Yang, H., Yuan, B., Zhang, X. \& Scherman, O. A. Supramolecular chemistry at interfaces: host-guest interactions for fabricating multifunctional biointerfaces. Acc. Chem. Res. 47, 2106-2115 (2014).

4. Szente, L. \& Szemán, J. Cyclodextrins in analytical chemistry: Host-guest type molecular recognition. Anal. Chem. 85, 8024-8030 (2013).

5. Harada, A., Kobayashi, R., Takashima, Y., Hashidzume, A. \& Yamaguchi, H. Macroscopic self-assembly through molecular recognition. Nat. Chem. 3, 34-37 (2011). 
6. Yu, G., Jie, K. \& Huang, F. Supramolecular amphiphiles based on host-guest molecular recognition motifs. Chem. Rev. 115, 7240-7303 (2015).

7. $\mathrm{Hu}$, Q.-D., Tang, G.-P. \& Chu, P. K. Cyclodextrin-based host-guest supramolecular nanoparticles for delivery: from design to applications. Acc. Chem. Res. 47, 2017-2025 (2014).

8. Zhang, J., Sun, H. \& Ma, P. X. Host-guest interaction mediated polymeric assemblies: multifunctional nanoparticles for drug and gene delivery. ACS Nano 4, 1049-1059 (2010).

9. Zan, M., Li, J., Luo, S. \& Ge, Z. Dual pH-triggered multistage drug delivery systems based on host-guest interaction-associated polymeric nanogels. Chem. Commun. 50, 7824-7827 (2014).

10. Vriezema, D. M., Aragonès, M. C., Elemans, J. A. A. W., Cornelissen, J. J. L. M., Rowan, A. E. and Nolte, R. J. M. Self-assembled nanoreactors. Chem. Rev. 105, 1445-1489 (2005).

11. Mirabaud, A., Mulatier, J.-C., Martinez, A., Dutasta, J.-P. \& Dufaud, V. Merging hostguest chemistry and organocatalysis for the chemical valorization of $\mathrm{CO}_{2}$. Catal. Today 281, 387-391 (2017).

12. Bolliger, J. L., Belenguer, A. M. \& Nitschke, J. R. Enantiopure water-soluble [Fe $\left.4 \mathrm{~L}_{6}\right]$ cages: host-guest chemistry and catalytic activity. Angew. Chemie Int. Ed. 52, 7958-7962 (2013).

13. Zhang, M., Ma, W. J., He, C. T., Jiang, L. \& Lu, T. B. Highly selective recognition and fluorescence imaging of adenosine polyphosphates in aqueous solution. Inorg. Chem. 52, 4873-4879 (2013).

14. Soylemez, S., Hacioglu, S. O., Kesik, M., Unay, H., Cirpan, A., Toppare, L. A novel and effective surface design: conducting polymer/ $\beta$-cyclodextrin host-guest system for cholesterol biosensor. ACS Appl. Mater. Interfaces 6, 18290-18300 (2014).

15. Xie, S., Zhang, J., Yuan, Y., Chai, Y. \& Yuan, R. An electrochemical peptide cleavagebased biosensor for prostate specific antigen detection via host-guest interaction between ferrocene and $\beta$-cyclodextrin. Chem. Commun. 51, 3387-3390 (2015).

16. Rudkevich, D. M. \& Leontiev, A. V. Molecular encapsulation of gases. Aust. J. Chem. 57, 713-722 (2004).

17. Kosaka, W., Yamagishi, K., Zhang, J. \& Miyasaka, H. Gate-opening gas adsorption and host-guest interacting gas trapping behavior of porous coordination polymers under applied AC electric fields. J. Am. Chem. Soc. 136, 12304-12313 (2014).

18. Gabard, J. \& Collet, A. Synthesis of a (D3)-bis(cyclotriveratrylenyl) macrocage by stereospecific replication of a (C3)-subunit. J. Chem. Soc. Chem. Commun. 1137-1139 (1981).

19. Collet, A. In comprehensive supramolecular chemistry. 2, (1996).

20. Brotin, T. \& Dutasta, J.-P. Cryptophanes and their complexes-present and future. Chem. Rev. 109, 88-130 (2009). 
21. Zhang, D., Martinez, A. \& Dutasta, J.-P. Emergence of hemicryptophanes: from synthesis to applications for recognition, molecular machines, and supramolecular catalysis. Chem. Rev. 117, 4900-4942 (2017).

22. Schmitt, A., Robert, V., Dutasta, J. \& Martinez, A. Synthesis of the first water-soluble hemicryptophane host: selective recognition of choline in aqueous medium. Org. Lett. 16, 2374-2377 (2014).

23. Dawei, Z., Gao, G., Guy, L. Robert, V., Dutasta, J.-P. and Martinez, A. A fluorescent heteroditopic hemicryptophane cage for the selective recognition of choline phosphate. Chem. Commun. 51, 2679-2682 (2015).

24. Perraud, O., Lefevre, S., Robert, V., Martinez, A. \& Dutasta, J.-P. Hemicryptophane host as efficient primary alkylammonium ion receptor. Org. Biomol. Chem. 10, 1056-1059 (2012).

25. Perraud, O., Martinez, A. \& Dutasta, J.-P. Exclusive enantioselective recognition of glucopyranosides by inherently chiral hemicryptophanes. Chem. Commun. (Camb). 47, 5861-5863 (2011).

26. Cochrane, J. R., Schmitt, A., Wille, U. \& Hutton, C. a. Synthesis of cyclic peptide hemicryptophanes: enantioselective recognition of a chiral zwitterionic guest. Chem. Commun. (Camb). 49, 8504-8506 (2013).

27. Perraud, O., Robert, V., Martinez, A. \& Dutasta, J. P. The cooperative effect in ion-pair recognition by a ditopic hemicryptophane host. Chem. - A Eur. J. 17, 4177-4182 (2011).

28. Makita, Y., Sugimoto, K., Furuyoshi, K., Ikeda, K., Fujiwara, S., Shin-ike, T., Ogawa, A. A Zinc(II)-included hemicryptophane: facile synthesis, characterization, and catalytic activity. Inorg. Chem. 49, 7220-7222 (2010).

29. Perraud, O., Robert, V., Martinez, A. \& Dutasta, J.-P. A designed cavity for zwitterionic species: selective recognition of taurine in aqueous media. Chem. - A Eur. J. 17, 1340513408 (2011).

30. Yamashita, M. \& Fenn, J. B. Electrospray ion source. Another variation on the free-jet theme. J. Phys. Chem. 88, 4451-4459 (1984).

31. Fenn, J. B., Mann, M., Meng, C. K., Wong, S. F. \& Whitehouse, C. M. Electrospray ionization for mass spectrometry of large biomolecules. Science (80-. ). 246, 64-71 (1989).

32. Fenn, J. B., Mann, M., Meng, C. K., Wong, S. F. \& Whitehouse, C. M. Electrospray ionization-principles and practice. Mass Spectrom. Rev. 9, 37-70 (1990).

33. Abdoul-carime, H., Farizon, B., Farizon, M., Mulatier, J.C., Dutasta, J.P., Chermette, H. Solution vs. gas phase relative stability of the choline/acetylcholine cavitand complexes. Phys. Chem. Chem. Phys. 17, 4448-4457 (2015).

34. Carroy, G., Lemaur, V., De Winter, J., Isaacs, L., De Pauw, E., Cornil, J. and Gerbaux, P. Energy-resolved collision-induced dissociation of non-covalent ions: charge- and guestdependence of decomplexation reaction efficiencies. Phys. Chem. Chem. Phys. 18, 12557-12568 (2016). 
35. Ma, X., Wei, Z., Xiong, X., Jiang, Y., He, J., Zhang, S., Fang, X. and Zhang, X. Gasphase fragmentation of host-guest complexes between $\beta$-cyclodextrin and small molecules. Talanta 93, 252-256 (2012).

36. Wang, L., Chai, Y., Sun, C. \& Armstrong, D. Complexation of cyclofrunctans with transition metal ions studied by electrospray ionization mass spectrometry and collisioninduced dissociation. J. Mass Spectrom. 323-324, 21-27 (2012).

37. Wei, W., Chu, Y., Wang, R., He, X. \& Ding, C. Quantifying non-covalent binding affinity using mass spectrometry: A systematic study on complexes of cyclodextrins with alkali metal cations. Rapid Commun. Mass Spectrom. 29, 927-936 (2015).

38. Armentrout, P. B. \& Baer, T. Gas-phase ion dynamics and chemistry. J. Phys. Chem. 100, 12866-12877 (1996).

39. Rodgers, M. \& Armentrout, P. Noncovalent metal-ligand bond energies as studied by threshold collision-induced dissociation. Mass Spectrom. Rev. 19, 215-247 (2000).

40. Armentrout, P. B. Mass spectrometry - Not just a structural tool: The use of guided ion beam tandem mass spectrometry to determine thermochemistry. J. Am. Soc. Mass Spectrom. 13, 419-434 (2002).

41. Gatineau, D., Memboeuf, A., Milet, A., Cole, R. B., Dossmann, H., Gimbert, Y. and Lesage, D. Experimental bond dissociation energies of benzylpyridinium thermometer ions determined by threshold-CID and RRKM modeling. Int. J. Mass Spectrom. 417, 6975 (2017).

42. Dunbar, R. C. BIRD (blackbody infrared radiative dissociation): evolution, principles, and applications. Mass Spectrom. Rev. 23, 127-158 (2004).

43. Price, W. D., Schnier, P. D. \& Williams, E. R. Tandem mass spectrometry of large biomolecule ions by blackbody infrared radiative dissociation. Anal. Chem. 68, 859-866 (1996).

44. Price, W. D., Schnier, P. D., Jockusch, R. A., Strittmatter, E. F. \& Williams, E. R. Unimolecular reaction kinetics in the high-pressure limit without collisions. J. Am. Chem. Soc. 118, 10640-10644 (1996).

45. McLuckeya, S. A., Wells, J. M., Stephenson, J. L. \& Goeringer, D. E. Novel quadrupole ion trap methods for characterizing the chemistry of gaseous macro-ions. Int. J. Mass Spectrom. 200, 137-161 (2000).

46. Butcher, D. J., Asano, K. G., Goeringer, D. E. \& McLuckey, S. a. Thermal dissociation of gaseous bradykinin ions. J. Phys. Chem. A 103, 8664-8671 (1999).

47. Asano, K. G., Goeringer, D. E. \& McLuckey, S. A. Thermal dissociation in the quadrupole ion trap: ions derived from leucine enkephalin. Int. J. Mass Spectrom. 185, 207-219 (1999).

48. Laskin, J. \& Futrell, J. H. Collisional activation of peptide ions in FT-ICR mass spectrometry. Mass Spectrom. Rev. 22, 158-181 (2003).

49. Laskin, J. \& Futrell, J. H. Activation of large lons in FT-ICR mass spectrometry. Mass Spectrom. Rev. 24, 135-167 (2005). 
50. Laskin, J., Denisov, E. \& Futrell, J. H. Fragmentation energetics of small peptides from multiple-collision activation and surface-induced dissociation in FT-ICR MS. Int. J. Mass Spectrom. 219, 189-201 (2002).

51. Zeisel, S. H. \& Blusztajn, J. K. Choline and human nutrition. Annu. Rev. Nutr. 14, 269 296 (1994).

52. Angus, J. A. \& Lew, M. J. Interpretation of the acetylcholine test of endothelial cell dysfunction in hypertension. J. Hypertens. 10, 179-186 (1992).

53. Millian, N. S. \& Garrow, T. a. Human betaine-homocysteine methyltransferase is a zinc metalloenzyme. Arch. Biochem. Biophys. 356, 93-98 (1998).

54. Finkelstein, J. D., Harris, B. J. \& Kyle, W. E. Methionine metabolism in mammals: Kinetic study of betaine-homocysteine methyltransferase. Arch. Biochem. Biophys. 153, 320-324 (1972).

55. Przybylski, C., Bonnet, V. \& Cézard, C. Probing the common alkali metal affinity of native and variously methylated $\beta$-cyclodextrins by combining electrospray-tandem mass spectrometry and molecular modeling. Phys. Chem. Chem. Phys. 17, 19288-305 (2015).

56. Lifshitz, C. Time-resolved appearance energies, breakdown graphs, and mass spectra: The elusive "kinetic shift". Mass Spectrom. Rev. 1, 309-348 (1982).

57. Lifshitz, C. Kinetic shifts. Eur. J. Mass Spectrom. 8, 85-98 (2002).

58. Rice, O. K. \& Ramsperger, H. C. Theories of unimolecular gas reactions at low pressures. J. Am. Chem. Soc. 49, 1617-1629 (1927).

59. Kassel, L. S. Studies in homogeneous gas reactions. I. J. Phys. Chem. 32, 225-242 (1928).

60. Marcus, R. a. \& Rice, O. K. The kinetics of the recombination of methyl radicals and iodine atoms. J. Phys. Chem. 55, 894-908 (1951).

61. Marcus, R. Unimolecular dissociations and free radical recombination reactions. J. Chem. Phys. 20, 359-364 (1952).

62. Perraud, O., Tommasino, J.B., Robert, V., Albela, B., Khrouz, L., Bonneviot, L., Dutasta, J.P. and Martinez, A. Hemicryptophane-assisted electron transfer: a structural and electronic study. Dalton Trans. 42, 1530-5 (2013).

63. Chatelet, B., Payet, E., Perraud, O., Dimitrov-Raytchev, P., Chapellet, L.L., Dufaud, V., Martinez, A. and Dutasta, J.P. Shorter and modular synthesis of hemicryptophane-tren derivatives. Org. Lett. 13, 3706-3709 (2011).

64. Ichou, F., Lesage, D., Machuron-Mandard, X., Junot, C., Cole, R.B., Tabet, J.-C. Collision cell pressure effect on CID spectra pattern using triple quadrupole instruments: a RRKM modeling. J. Mass Spectrom. 48, 179-186 (2013).

65. Schnier, P. D., Price, W. D., Strittmatter, E. F. \& Williams, E. R. Dissociation energetics and mechanism of leucine enkephalin $(\mathrm{M}+\mathrm{H})+$ and $(2 \mathrm{M}+\mathrm{X})+$ ions $(\mathrm{X}=\mathrm{H}, \mathrm{Li}, \mathrm{Na}, \mathrm{K}$, and $\mathrm{Rb}$ ) measured by blackbody infrared radiative dissociation. J. Am. Soc. Mass Spectrom. 8, 771-780 (1997). 
66. Schmidt, M. W., Baldridge, K. K., Boatz, J. A., Elbert, S.T., Gordon, M. S., Jensen, J. H., Koseki, S., Matsunaga, N., Nguyen, K. A., Su, S., Windus, T. L., Dupuis, M. and Jr, J. A. M. General atomic and molecular electronic structure system. J. Comput. Chem. 14, 1347-1363 (1993).

67. Gordon, M. S. \& Schmidt, M. W. Advances in electronic structure theory: GAMESS adecade later. Theory and Applications of Computational Chemistry: The First Forty Years (2005).

68. Derrick, P. J., Loyd, P. M. \& Christie, J. R. Physical chemistry of ion reactions in advanced mass spectrometry, vol. 13. (Wiley: Chichester, UK, 1995).

69. Moon, J. H., Oh, J. Y. \& Kim, M. S. A systematic and efficient method to estimate the vibrational frequencies of linear peptide and protein ions with any amino acid sequence for the calculation of Rice-Ramsperger-Kassel-Marcus rate constant. J. Am. Soc. Mass Spectrom. 17, 1749-1757 (2006).

70. L. Drahos and V. Karoly. MassKinetics: a theoretical model of mass spectra incorporating physical processes, reaction kinetics and mathematical descriptions aszí o Drahos and aroly ekey. J. Mass Spectrom 36, 237-263 (2001).

71. Naban-Maillet, J., Lesage, D., Bossée, A., Gimbert, Y., Sztáray, J., Vékey, K and Tabet, J.-C. Internal energy distribution in electrospray ionization. J. Mass Spectrom. 40, 1-8 (2005).

72. Pak, A., Lesage, D., Gimbert, Y., Vékey, K. \& Tabet, J. C. Internal energy distribution of peptides in electrospray ionization: ESI and collision-induced dissociation spectra calculation. J. Mass Spectrom. 43, 447-455 (2008).

73. Ichou, F., Schwarzenberg, A., Lesage, D., Alves, S., Junot, C., Machuron-Mandard, X. and Tabet, J.C. Comparison of the activation time effects and the internal energy distributions for the CID, PQD and HCD excitation modes. J. Mass Spectrom. 49, 498508 (2014).

74. Chen, P. Electrospray ionization tandem mass spectrometry in high-throughput screening of homogeneous catalysts. Angew. Chemie Int. Ed. 42, 2832-2847 (2003).

75. Lesage, D., Blu, J., Tabet, J. C. \& Gimbert, Y. Intermolecular versus intramolecular Pauson-Khand reaction in gas phase: Limitation and feasibility. J. Organomet. Chem. 809, 7-13 (2016).

76. Vékey, K. Internal energy effects in mass spectrometry. J. Mass Spectrom. 31, 445-463 (1996). 
GRAPHICAL ABSTRACT

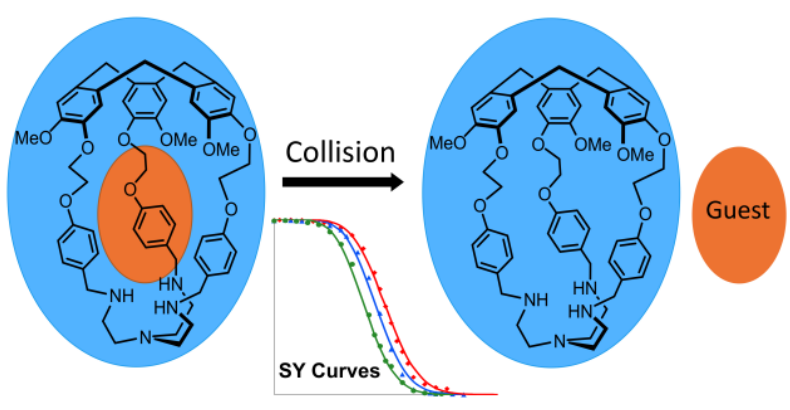

